\title{
The effects of optical sensor-tissue separation in endocavitary photoplethysmography
}

To cite this article before publication: Zaibaa Patel et al 2018 Physiol. Meas. in press https://doi.org/10.1088/1361-6579/aacc1d

\section{Manuscript version: Accepted Manuscript}

Accepted Manuscript is "the version of the article accepted for publication including all changes made as a result of the peer review process, and which may also include the addition to the article by IOP Publishing of a header, an article ID, a cover sheet and/or an 'Accepted

Manuscript' watermark, but excluding any other editing, typesetting or other changes made by IOP Publishing and/or its licensors"

This Accepted Manuscript is (c) 2018 Institute of Physics and Engineering in Medicine.

During the embargo period (the 12 month period from the publication of the Version of Record of this article), the Accepted Manuscript is fully protected by copyright and cannot be reused or reposted elsewhere.

As the Version of Record of this article is going to be / has been published on a subscription basis, this Accepted Manuscript is available for reuse under a CC BY-NC-ND 3.0 licence after the 12 month embargo period.

After the embargo period, everyone is permitted to use copy and redistribute this article for non-commercial purposes only, provided that they adhere to all the terms of the licence https://creativecommons.org/licences/by-nc-nd/3.0

Although reasonable endeavours have been taken to obtain all necessary permissions from third parties to include their copyrighted content within this article, their full citation and copyright line may not be present in this Accepted Manuscript version. Before using any content from this article, please refer to the Version of Record on IOPscience once published for full citation and copyright details, as permissions will likely be required. All third party content is fully copyright protected, unless specifically stated otherwise in the figure caption in the Version of Record.

View the article online for updates and enhancements. 


\begin{abstract}
.
Objective: Intestinal anastomotic failure that occurs mainly due to ischaemia is a serious risk in colorectal cancer patients undergoing surgery. Surgeons continue to rely on subjective methods such as visual inspection to assess intestinal viability during surgery and there are no clinical tools to directly monitor viability postoperatively. A dual wavelength, reflectance optical sensor has been developed for continuous and dynamic monitoring of intestinal viability via the intestinal lumen. Maintaining direct contact between the sensor and the inner intestinal wall can be difficult in an intraluminal design, therefore impacting on signal acquisition and quality. This paper investigates the effect of direct contact versus variable distances between the sensor and the tissue surface of the buccal mucosa as a surrogate.

Approach: The in-vivo study involved 20 healthy volunteers to measure the effect of optical sensor-tissue distances on the ability to acquire photoplethysmography signals and their quality. Signals were acquired from the buccal mucosa at five optical sensor-tissue distances.

Main results: Distances between $0 \mathrm{~mm}$ (contact) to $5 \mathrm{~mm}$ were the most optimal, producing signals of high quality and signal-to-noise ratio, resulting in reliable estimations of the blood oxygen saturation. Distances exceeding $5 \mathrm{~mm}$ compromised the acquired signals, and were of poor quality, thereby unreliably estimating the blood oxygen saturation.

Significance: The developed optical sensor proved to be reliable for acquiring photoplethysmography signals for cases where distances between the optical sensor-tissue may arise during the assessment of intraluminal intestinal viability.
\end{abstract}

Keywords: Photoplethysmography, pulse oximetry, optical sensor, intestine, viability, physiological monitoring.

Submitted to: Physiol. Meas. (SECOND SUBMISSION - AMENDMENT)




\section{Introduction}

Colorectal cancer is the fourth most common cause of cancer deaths in the UK (Cancer Research, 2016) and surgery is the only curative treatment. Surgery for colorectal cancer often involves excision of the tumour bearing intestine followed by an intestinal anastomosis to restore intestinal/continuity. Restoring intestinal continuity after cancer resection is deemed the gold standard as any stoma after surgery is proven to cause a poor quality of life in cancer survivors. In $2-10 \%$ of patients anastomotic failure occurs mainly due to inadequate vascularity surrounding the anastomosis (Jafari et al., 2013). Currently, there are no reliable tools to directly monitor intestinal perfusion across a newly constructed anastomosis and hence, prediction of anastomotic failure remains a clinical challenge (Jafari et al., 2013).

Splanchnic circulation is the main source of blood supply to the intestinal tract which is susceptible to ischemia and is easily compromised to a variable extent in systemic illnesses (Nachiappan et al., 2014). Intestinal ischaemia is a cause of disruption of gut-mucosal barrier, which allows bacterial translocation and endotoxin absorption into the portal circulation. Consequently, there is amplification of systemic inflammatory response (Hickey et al., 2010).

The current gold standards to assess the adequacy of intestinal viability intraoperatively are based on visual inspection of the colour, arterial pulsation and presence of peristalsis (Kingham et al., 2009). The majority of the other techniques previously described for assessing the intestinal viability via measurements of oxygen tension (Lee et al., 2006), fluorescence (Jafári et al., 2015) and laser Doppler flowmetry (Abdollahi et al., 2015) have all remained as research tools and have not been adopted into mainstream clinical practice. Significantly, none of the described techniques allow continuous and dynamic monitoring of intestinal viability especially in the postoperative period (Hyman, 2009). Additionally, most researched technologies are operator dependent, resulting in inter-observer inconsistency and unreliable technological performance.

To overcome these limitations, a novel intraluminal optical sensor system was proposed (Patel et al., 2017). The intraluminal sensor system bases its technology on photoplethysmography (PPG) and pulse oximetry (PO), which is known for many clinical and research applications (Kyriacou, 2006). The intraluminal PPG sensor system aims to provide dynamic monitoring of intestinal viability through a continuous measurement of blood volumetric changes through PPG amplitudes and perfusion indexes. Thereby, enabling a potential clinical monitoring tool for both intraoperative and postoperative use.

The PPG sensor is inserted into the lumen of the intestine via the anal orifice. Light emitting from the sensor at two wavelengths, red and infrared, penetrates the intestinal mucosal lining. The reflected light intensity is measured by the photodetector, where changes in blood volume and oxygen saturation $\left(\mathrm{S}_{p} \mathrm{O}_{2}\right)$ are estimated (Webster, 1997).

Conventionally, the acquisition of good quality PPG signals and the accuracy of $S_{p} O_{2}$ s depend on a reliable contact between the optical sensor and the tissue surface (Sun and Thakor, 2016; Allen, 2007; Hickey et al., 2011). However, in an intraluminal design the reliability of sensor-tissue surface contact is uncertain. Balloon designs, although might improve sensor-tissue surface contact might inadvertently cause pressure-related complications. Therefore, this study investigates the influence of direct contact versus no contact between the sensor and the tissue surface on the acquisition and quality of endocavitary 
photoplethysmograph signals. Furthermore, the study would provide information on the optimal distance in order to obtain reliable, high-quality signals.

Buccal mucosa is selected as a surrogate for intestinal mucosa due to its ease of access and tissué similarity. Using a custom made intraluminal probe, PPGs at two wavelengths were investigated. The paper aims to describe the technical developments of the dual wavelength intraluminal PPG probe and relevant instrumentation, and investigate the effects of varying optical sensor-tissue separations on PPG signals acquired from the buccal mucosa.

\section{Materials and methods}

A dual wavelength reflectance PPG sensor probe was developed to measure intestinal viability through an intraluminal route. Prior to its validation in the human intestine, evaluations were designed for obtaining measurements using the sensor from the human buccal mucosa, given the locations tissue similarity and its easy accessibility. A previous study reported successful PPG acquisition from the buccal mucosa of healthy human volunteers (Patel et al., 2017). The present study investigated the effects of varying the separation distance between the optical sensor and the tissue mucosa. Figure 1 illustrates the method in obtaining PPG signals when separation distances between the intraluminal PPG sensor and the buccal mucosa tissue were created.



Figure 1: Simplified representation of the signal acquisition method. The intraluminal PPG sensor (blue) records photoplethysmography signals at several distances, $x_{i}$, from the buccal mucosa. Let $x_{1}$ be $0 \mathrm{~mm}$ from the buccal mucosa. Initial photoplethysmography signals would be acquired at $x_{1}$ for a chosen measurement period, before increasing the sensor-buccal distance to $x_{2}, x_{3}$ till $x_{n}$.

\subsection{Intraluminal PPG sensor}

A prototype intraluminal PPG sensor has been developed and described (Patel et al., 2017). The capsular prototype, having a diameter and length of $5.5 \mathrm{~mm}$ and $10.2 \mathrm{~mm}$ respectively, was deemed unsuitable for intraluminal insertion into the human colon; therefore a second sensor of a longer length has been designed and fabricated for intraluminal colonic measurements, to reduce strain between the wire and sensor casing. 
A sensor printed circuit board (PCB), figure $2(\mathrm{a}-\mathrm{b})$, with the dimensions of $22 \times 4.2 \times 2.51 \mathrm{~mm}$ $(l \times w \times h)$ was fabricated using an electronic computer aided design software. The intraluminal PPG sensor consists of a red and infrared surface mount light emitting diodes (LEDs), with peak wavelength emissions of $660 \mathrm{~nm}$ (KP-2012SRC, Kingbright, Taiwan) and $880 \mathrm{~nm}$ (KP-2012SF4C, Kingbright, Taiwan) respectively. For the detection of back scattering of light from the tissue, a flat-top photodiode with an active area of $0.65 \mathrm{~mm}^{2}$ and peak wavelength sensitivity of $900 \mathrm{~nm}$ (SR10BP, Excelitas technologies, Massachusetts) was used. The separation distance from the centre of the photodiode to the centre of each LED was $5 \mathrm{~mm}$. Previous studies have shown such separation distance would provide good quality PPGs with a high signal-to-noise ratio (SNR) (Hickey and Kyriacou, 2007).



(a)



Figure 2: Mechanical drawing with dimensions of the components required to develop the modified intraluminal PPG sensor. Drawings illustrate: (a) reflectance PPG sensor (side view), where the emitterdetector separation distance and the length and total height are annotated; (b) reflectance PPG sensor (top view), indicating the width and location of the components, such as the light emitting diodes and the photodetector. (c) sensor casing dimensions, where the rectangular recess is of the same length of the reflectance PPG sensor, allowing the placement of the reflectance PPG sensor within the capsular casing.

\section{Sensor casing}

The anatomical structures of the large intestine (colon and rectum) were taken into consideration when developing the intraluminal PPG sensor. The large intestine is tubular shaped, with an approximate lumen dimension and wall thickness of $50 \mathrm{~mm}$ and $5 \mathrm{~mm}$ (Nicholls and Dozois, 1997).

A capsular design for the casing was developed using SolidWorks 2016 (Dassault Systems, VlizyVillacoublay, FR) and 3D printing technology, Object24 3D printer (Stratasys Ltd, Minnesota, US). The casing was designed to provide smooth and even surfaces with rounded edges to minimise any intestinal damage during the placement of the sensor. The modified sensor casing, shown in figure 2(c), has a diameter and length of $6 \times 36 \mathrm{~mm}$ respectively, with a rectangular recess, allowing placement of the reflectance $\mathrm{PPG}$ sensor within the capsular casing.

The reflectance PPG sensor was securely fixed within the sensor casing, with black absorbing material surrounding the optics to reduce the effect of optical shunt (penumbra effect). An optically clear medical epoxy (Dymax Corporation, Torrington, CT), was used to seal the reflectance PPG sensor within the casing to provide electrical isolation. Electrical isolation was imperative, should the sensor come into contact with biological tissue and fluids. A multicore cable drawn through the sensor casing and soldered 
onto the PCB, provides electrical contact between the intraluminal PPG sensor to the processing and data acquisition system. To allow detected signals to enter the processing and data acquisition system, a male D-Sub 9 connector was used (figure 3).

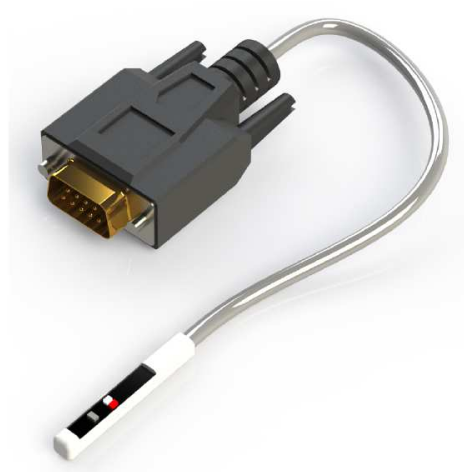

(a)



(b)

Figure 3: The intraluminal photoplethysmography sensor for the assessment of intestinal viability. (a) 3D visualisation of the intraluminal PPG sensor; (b) Image of the developed intraluminal sensor. A black absorbing material surrounds the optics, acting as an optical barrier before being sealed with a medical epoxy. The sensor includes a D-Sub 9 connector allowing connection capabilities with the processing and data acquisition system.

\subsection{Development of an adjustable distance apparatus}

As figure 1 suggests, the study required an apparatus to alter the optical sensor-tissue separation distance in a controlled and repeatable manner. Five optical sensor-tissue separations were chosen for the study; $0 \mathrm{~mm}, 2.3 \mathrm{~mm}, 8 \mathrm{~mm}$ and $10 \mathrm{~mm}$. At each separation, the sensor was inserted into the mouth and positioned in the maxillary vestibule, directing the emitted light into the inner cheek buccal mucosal lining.

Measurements at the optical sensor-tissue distance of $0 \mathrm{~mm}$ (contact) required the sensor to be covered in a clean plastic sheath. Measurements with the use of the plastic sheath was considered as direct contact between the intraluminal PPG sensor and the buccal mucosal lining, with the assumption of the sheath being of negligible thickness. The remaining measurements utilised customised clean, clear rectal catheters (Pennine Healthcare, Derby, UK), with inner dimensions of $13.2 \mathrm{~mm}$ and the length of $40 \mathrm{~mm}$. The purpose of the catheters was to obtain optical sensor-tissue distances as described above. The first non-contact separation was $2.3 \mathrm{~mm}$, which corresponds to the wall thickness of the catheter.

In order to adjust the distances within the catheter, catheter caps were designed using SolidWorks 2016 (Dassault Systems, Vlizy-Villacoublay, FR). Each catheter cap was designed to ensure the sensor was accurately positioned at the needed distance. The portion of the cap that resides within the catheter (inner catheter cap) was designed to have the same dimension as the inner dimensions of the catheter. Figure 4(a) illustrates the recess position changing depending on the distance required. To guarantee the intraluminal PPG sensor directed towards the buccal mucosal lining at all times, a flat indent to 
(a)

$0 \mathrm{~mm}$

\section{$2.3 \mathrm{~mm}$}

(b)
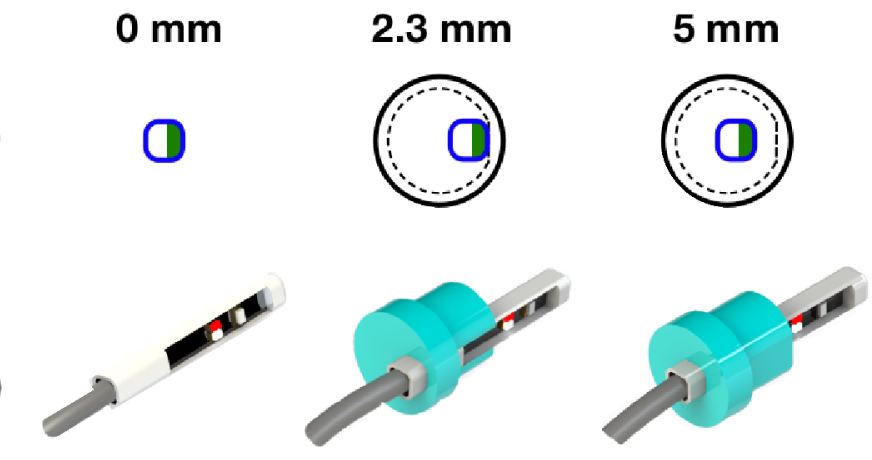

(c)

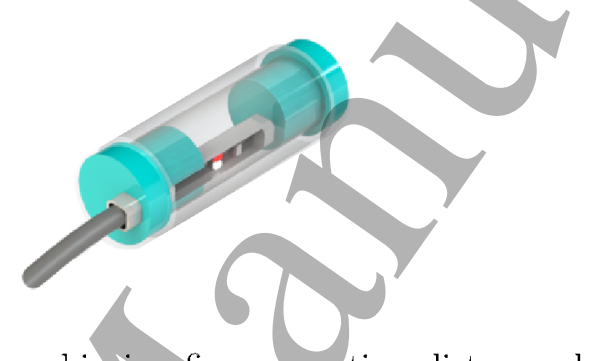

Figure 4: Adjustable distance apparatus achieving five separation distances between the intraluminal PPG sensor and the buccal mucosa. (a) Mechanical drawing of the apparatus, where all measurements, except at $0 \mathrm{~mm}$, requires the use of catheters and catheter caps. The inner cap $(\cdots)$ corresponds to the measurement distance of $2.3 \mathrm{~mm}$. (b) 3D visualisation of the intraluminal PPG sensor fitting into the extruded catheter caps, where the flat indent indicates the sensor direction. (c) Example of the full assembly for measurements at $2.3 \mathrm{~mm}$, where the intraluminal PPG sensor is inserted within the customised catheter and both catheter caps, extruded and sealed, placed on either ends of the catheter.

the catheter cap was created indicating the direction required for the sensor, as shown in figure $4(\mathrm{a}-\mathrm{b})$. The further the sensor moves away from the flat indent, the optical sensor-tissue distance increases. A recess within the catheter cap was designed to have the same dimensions of the intraluminal PPG sensor, ensuring the sensor would fit precisely, preventing movement or rotation within the catheter. Additional to the extruded catheter caps, sealed catheter caps were developed to avoid liquid ingress, as shown in figure $4(\mathrm{c})$.

\subsection{PPG processing and data acquisition system}

A battery operated, customised/PPG processing system, ZenPPG, developed by the Research Centre for Biomedical Engineering at City, University of London was utilised (Budidha et al., 2018). ZenPPG is a modular system of dual channels, permitting the acquisition of raw, dual wavelength PPG signals prior to analogue-to-digital conversion for offline analysis and further signal analysis. Both channels allow $\mathrm{PPG}$ acquisitions via the intraluminal optical sensor and an electrically and optically identical finger sensor.

Within a rectangular portable unit $(16.0 \times 10.3 \times 5.6 \mathrm{~cm})$, there are identical dual pulse oximeter channels, comprising of multiplexed current sources to drive the infrared and red LEDs, alternatively.

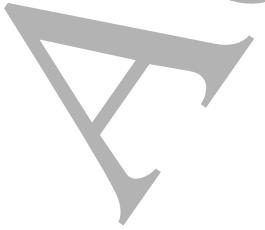


Back Panel

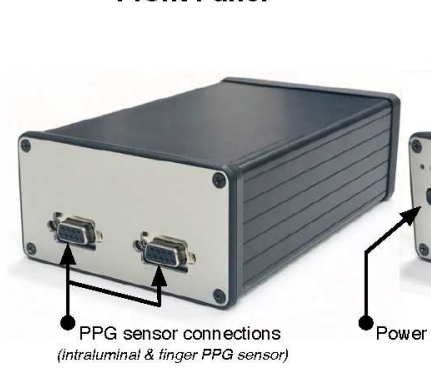

(a)

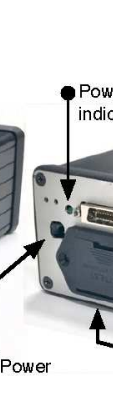

计

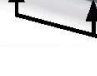

(b)

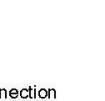

Instrumentation modules

\section{x}

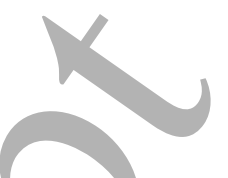
nce



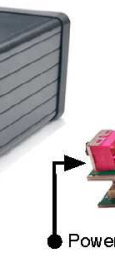

(b)

Figure 5: Photographs of the modular photoplethysmography processing and data acquisition system, ZenPPG. The enclosed system allows (a) connections of the intraluminal and finger PPG sensor on the front panel; (b) connections for the data acquisition and battery replacements; (c) ZenPPG instrumentation within the unit responsible for PPG processing. Modular system allows additional modules to be interconnected via a double-sided system bus, such as the vital signs module (not used in this study).

The current source module allows current for one channel to be adjusted digitally, whereas the second channel is altered through trimmers. When the LEDs illuminate the tissue, the light detected from the photodiode are passed to a trans-impedance amplifier for current-to-voltage conversion. The core board of the ZenPPG was accountable for the multiplexing and de-multiplexing process for the dual channels via a micro-controller (Atmel ATtiny 2313-20SU, California, US). Additionally, signals were passed through filters to condition the acquired signals preceding digitisation.

All output signals were digitised by a 16-bit data acquisition card (NI DAQ-6211, National Instruments Corp., Texas, US), at a sampling rate of $1 \mathrm{kHz}$. With the use of LabVIEW (National Instruments Corp., Texas, US), all signals were saved and displayed simultaneously, with the availability of adjusting the light intensities, by digitally controlling the driving currents of the LEDs (Budidha et al., 2018).

\section{$2.4 \quad$ In-vivo volunteer study}

\section{Subjects}

With institutional research ethics approval, 20 healthy volunteers were recruited. The healthy volunteers were equally distributed between males and females, with ages ranging from 18 to 38 (mean age $[ \pm S D]: 28$ $[ \pm 4]$ ) years. Following full explanation of the study objective, informed consent was obtained from all subjects prior to the commencement of the study.

\section{Study protocol}

The in-vivo study was carried out in the Research Centre for Biomedical Engineering Physiological Measurements laboratory. The room temperature was maintained at an average temperature of $20 \pm$ $2{ }^{\circ} \mathrm{C}$ throughout the study. Overhead fluorescent lights were switched off to eliminate additional light interference when recordings took place. To minimise motion artefacts, volunteers rested comfortably 
in a semi-Fowlers position, which is the supine position with the head of the bed at an upright angle between $30-40^{\circ}$. Furthermore, all reusable materials were disinfected using sterilising fluid (Milton, $U K$ ) and volunteers were offered to rinse their mouth with mouthwash, before and after the study.

Two probes were utilised concurrently, the intraluminal PPG sensor and a finger reflectance PPG sensor for buccal mucosa and finger measurements respectively. Measurements from both locations were obtained at five sensor-tissue separations, $0 \mathrm{~mm}, 2.3 \mathrm{~mm}, 5 \mathrm{~mm}, 8 \mathrm{~mm}$ and $10 \mathrm{~mm}$. For mucosal measurements to commence at $0 \mathrm{~mm}$, the intraluminal PPG sensor was covered in a plastic sheath. The covered sensor was inserted into the mouth and positioned in the maxillary vestibule, directing towards the left cheek buccal mucosa. Subsequently, the separation distance between the sensor and the mucosal lining was achieved with the use of the adjustable distance apparatus (section 2.2). After positioning the sensor correctly in the maxillary vestibule, volunteers were asked to close their mouth. All raw measurements were acquired at two light intensities, by driving the LEDs simultaneously with currents of $20 \mathrm{~mA}$ and $40 \mathrm{~mA}$, respectively. Two minutes of recorded data at each distance was saved and displayed on a computer running LabVIEW. The overall study duration lasted for up to 45 minutes, where short breaks were provided between changes of the sensor-tissue separations and the driving currents of the LEDs.

\section{Data analysis and statistics}

The recorded PPG signals were processed and analysed offline using Matlab ${ }^{\circledR}$. The recorded PPG signals were down sampled to $100 \mathrm{~Hz}$ to remove high frequency noise. Further noise reduction and DC offsets were removed with digital filters. A band-pass, finite impulse response (FIR) filter, with a pass-band between $0.45-10 \mathrm{~Hz}$ and $60 \mathrm{~dB}$ attenuation in the stop-band was designed. The designed filter coefficients were utilised with filtfilt (Mitra, 2001) in Matlab ${ }^{\circledR}$, to perform zero-phase filtering of all signals.

For each volunteer, 20 second samples of the PPG signals were selected from the recorded PPG signals, from the buccal mucosa and finger at the five sensor-tissue separations at each light intensity. From the PPG signal, the changes in absorbance of the arterial blood (ac component) can be identified from the non-pulsatile venous blood and other body tissue (dc component) due to the pulsatile nature of the arterial blood (Hickey and Kyriacou, 2007). To identify each component of both red and infrared, a 2 second rolling window over the chosen 20 second measurement period was used to obtain the peak-to-peak amplitudes of the pulsatile signal (ac) and the relatively constant values of light attenuation (dc).

To aid in comparisons, let the term 'overall mean and standard deviation' $\left(\bar{M}_{q} \pm \overline{S D}_{q}\right)$ be the mean of the means of the signal parameter of interest $(q)$ from all subjects. Therefore, for a signal parameter $(q)$, first the mean per subject are obtained; followed by an average of all the subjects' mean $\left(\bar{M}_{q}\right)$ and standard deviation $\left( \pm \overline{S D}_{q}\right)$.

Comparison of the ac PPG amplitude changes against the sensor-tissue separation were made, where the overall mean and standard deviation $\left(\bar{M}_{a c} \pm \overline{S D}_{a c}\right)$ was calculated for each of the five separations, when the LEDs were driven with currents of $20 \mathrm{~mA}$ and $40 \mathrm{~mA}$, respectively.

The quality of the acquired ac PPG signals was assessed by measuring the signal-to-noise ratio (SNR). This was achieved by using measurements of the signal against background noise in the time domain with the usage of MATLAB ${ }^{\circledR}$ 's robust quadratic regression and snr functions. For comparisons, the overall mean of the SNR of the ac PPG signals $\left(\bar{M}_{S N R}\right)$ were obtained for each of the five separations and the 
two LED driving currents.

For the estimation of the arterial oxygen saturation, $S_{p} O_{2}$, the estimation followed two steps: calculating the ratio of the components (i.e. ratio of ratios, $R_{O S}$ ) at two wavelengths, equation 1 and correlating the ratio of ratios to $\mathrm{S}_{p} \mathrm{O}_{2}$, equation 2 (Kyriacou, 2013). Similarly, the comparisons of $\mathrm{S}_{p} \mathrm{O}_{2}$ against the sensor-tissue separation were obtained by calculating the overall mean and standard deviation $\left(\bar{M}_{S_{p} O_{2}} \pm \overline{S D}_{S_{p} O_{2}}\right)$ for each of the five separations and the two LED driving currents.

$$
\begin{gathered}
R_{O S}=\frac{\left(\frac{A C}{D C}\right)_{R}}{\left(\frac{A C}{D C}\right)_{I R}} \\
S_{p} O_{2}=110-25\left(R_{O S}\right)
\end{gathered}
$$

The Kolmogorov-Smirnov (KS) test (Massey, 1951), was used to validate normality of the ac PPG signals from the buccal mucosa and finger. For multiple comparisons, the one-way analysis of variance (ANOVA) (Hogg and Ledolter, 1987) was then applied, to test for statistical significance between the ac PPG amplitudes means acquired at the recorded distances. If significant difference were found, a post-hoc correction method, Bonferroni, was used (Neter et al., 1996). P-values less than 0.05 were considered to be statistically significant.

\section{Results}

Analysis of the recorded PPG signals was performed on 19 of the 20 volunteers. In one volunteer, the recorded signals were particularly hindered due to electromyogram interference from the cheek and overall motion artefact. Identifying fundamental characteristics of the PPG waveform was challenging, therefore this data set was excluded from further analysis.

PPG signals were recorded $(n=19)$, from both wavelengths and all separations between the intraluminal PPG sensor and the buccal mucosa lining. Figure 6 depicts typical infrared ac PPG traces from the buccal mucosal at each sensor-tissue distance, at two light intensities, utilising LED driving currents of $20 \mathrm{~mA}$ and $40 \mathrm{~mA}$, respectively. For illustration purposes, figure 6(c) depicts an example of noisy PPG signals obtained at a sensor-buccal distance of $10 \mathrm{~mm}$, when the LEDs driving currents were set to $20 \mathrm{~mA}$.

For each sensor-tissue distance, the mean infrared and red ac PPG amplitudes were calculated by averaging the amplitudes with the use of a 2 second rolling window over the chosen 20 second measurement period, section 2.4. The overall mean and standard deviation of the ac PPG amplitude $\left(\bar{M}_{a c} \pm \overline{S D}_{a c}\right)$ from the buccal mucosa for different sensor-buccal distances are illustrated in figure 7 for each light intensity where the LEDDs were driven with $20 \mathrm{~mA}$ and $40 \mathrm{~mA}$.

As described in section 2.4, the SNR was calculated. Figure 8 presents the overall mean of the SNR $\left(\bar{M}_{S N R}\right)$ for red and infrared ac PPG signals from the buccal mucosa at each sensor-buccal separation. The SNR was calculated for each wavelength when driven with currents of $20 \mathrm{~mA}$ and $40 \mathrm{~mA}$ per sensorbuceal separation.

Preliminary estimations of $\mathrm{S}_{p} \mathrm{O}_{2}$ was calculated using equation 1 and 2 respectively. Calculations were based on each subject per sensor-tissue separation from the buccal mucosa and finger at the two light intensities, where the LEDs were driven with currents of $20 \mathrm{~mA}$ and $40 \mathrm{~mA}$. 
(a) LED driving current: $20 \mathrm{~mA}$



(b) LED driving current: $40 \mathrm{~mA}$

\section{${ }^{110}$}

90

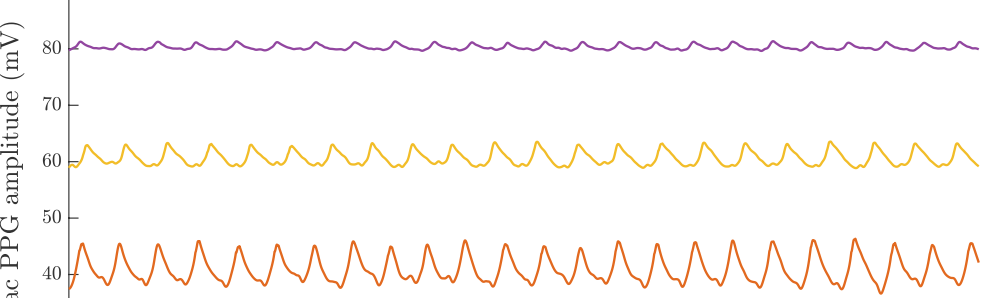
${ }^{20} \wedge N \wedge N A N A N A N A N A N A N A N A N$ $\begin{array}{rrrrrrrrrr}10 & 1 & 1 & 1 & 1 & 1 & 1 & 1 & 1 & 1 \\ 0 & 2 & 4 & 6 & 8 & 10 & 12 & 14 & 16 & 18\end{array}$

(c) Example of noisy signals

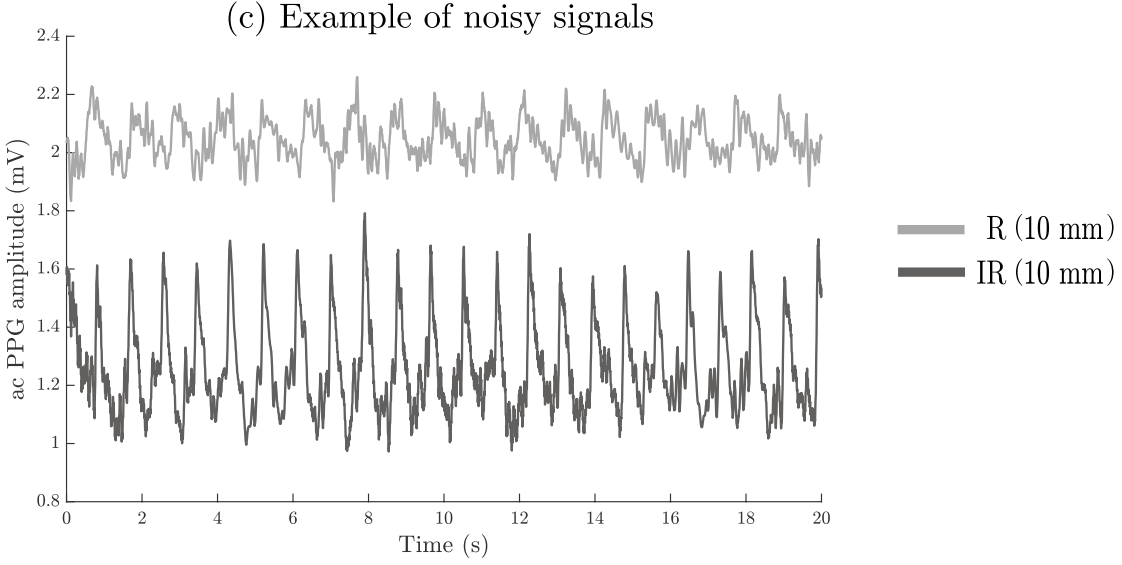

Figure 6: Twenty seconds of typical infrared ac PPG traces from a healthy volunteer at different sensor distances from the buccal mucosa. For visualisation purposes, five signals are equally spaced with a $20 \mathrm{mV}$ offset; as the offset increases, the separation increases. PPG measurements were obtained at two light intensities by driving the LEDs with currents of (a) $20 \mathrm{~mA}$ and (b) $40 \mathrm{~mA}$. (c) illustrates examples of noisy ac PPG signals from both wavelenths, infrared (bottom) and red (top). These were obtained at a separation of $10 \mathrm{~mm}$, where the LEDs driving current was $20 \mathrm{~mA}$. The two signals are equally spaced with a $1 \mathrm{mV}$ offset. 
Table 1: Overall mean and standard deviation of blood oxygen saturation $\left(\bar{M}_{S_{p} \mathrm{O}_{2}} \pm \overline{S D}_{S_{p} O_{2}}\right)$ estimated at each sensor-tissue separation. The results present the overall mean and standard deviation of blood oxygen saturation from the buccal mucosa and left middle finger; each obtained at the five distances.

\begin{tabular}{ccc}
\hline \hline & & \\
Distance $(\mathrm{mm})$ & \multicolumn{3}{c}{$\bar{M}_{S_{p} \mathrm{O}_{2}} \pm \overline{S D}_{S_{p} \mathrm{O}_{2}}(\%)$} \\
& Buccal & Finger \\
\hline 0 & $96 \pm 3$ & $97 \pm 1$ \\
2.3 & $98 \pm 1$ & $96 \pm 2$ \\
5 & $97 \pm 2$ & $96 \pm 2$ \\
8 & $96 \pm 4$ & $94 \pm 4$ \\
10 & $95 \pm 3$ & $94 \pm 5$ \\
\hline \hline
\end{tabular}

Table 2: One-way analysis of variance (ANOVA) tested for statistical significance between the overall mean ac photoplethysmography signals $\left(\bar{M}_{a c}\right)$ from two related sensor-buccal distance. A post-hoc comparison test, Bonferroni, was used to determine where statistical significance were found. The table presents the p-values of the paired test on each pair (first and second column). Bonferroni correction was tested at two light intensities by driving the LEDs with currents of $20 \mathrm{~mA}$ and $40 \mathrm{~mA}$. Tests where the null hypothesis could not be rejected are highlighted in red.

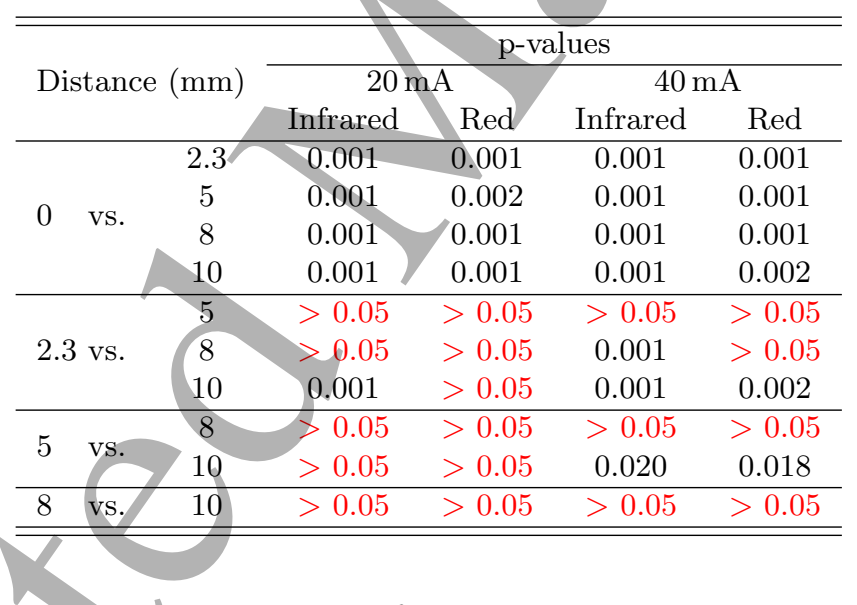

The $\bar{M}_{S_{p} O_{2}}$ values presented in table 1 were found to have a normal distribution (KS test) at each separation and hence, the t-test was used to determine significant differences between the $\bar{M}_{S_{p} O_{2}}$ from the buccal mucosa and finger per sensor-tissue distance. At each sensor-tissue separation, no significance was found, where all p-values were above 0.05 .

The $\bar{M}_{a c}$ from the buccal mucosa had a normal distribution (KS test) and hence, a one-way analysis of variance (ANOVA) tested for statistical significance between the $\bar{M}_{a c}$ from the buccal mucosa from two related sensor-buccal distance, for example, $8 \mathrm{~mm}$ versus $10 \mathrm{~mm}$ sensor-tissue distance. As significant differences were found, a post-hoc Bonferroni correction was used. Table 2 is a multiple comparisons table presenting the p-values from the Bonferroni correction of the paired tests on each sensor-buccal distance pair. The Bonferroni correction was tested at the two light intensities, by driving the LEDs with currents of $20 \mathrm{~mA}$ and $40 \mathrm{~mA}$. Tests that were unable to reject the null hypothesis are highlighted in red. 

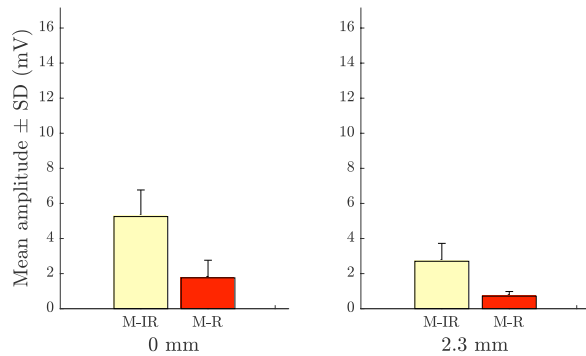

(a) LED driving current: $20 \mathrm{~mA}$
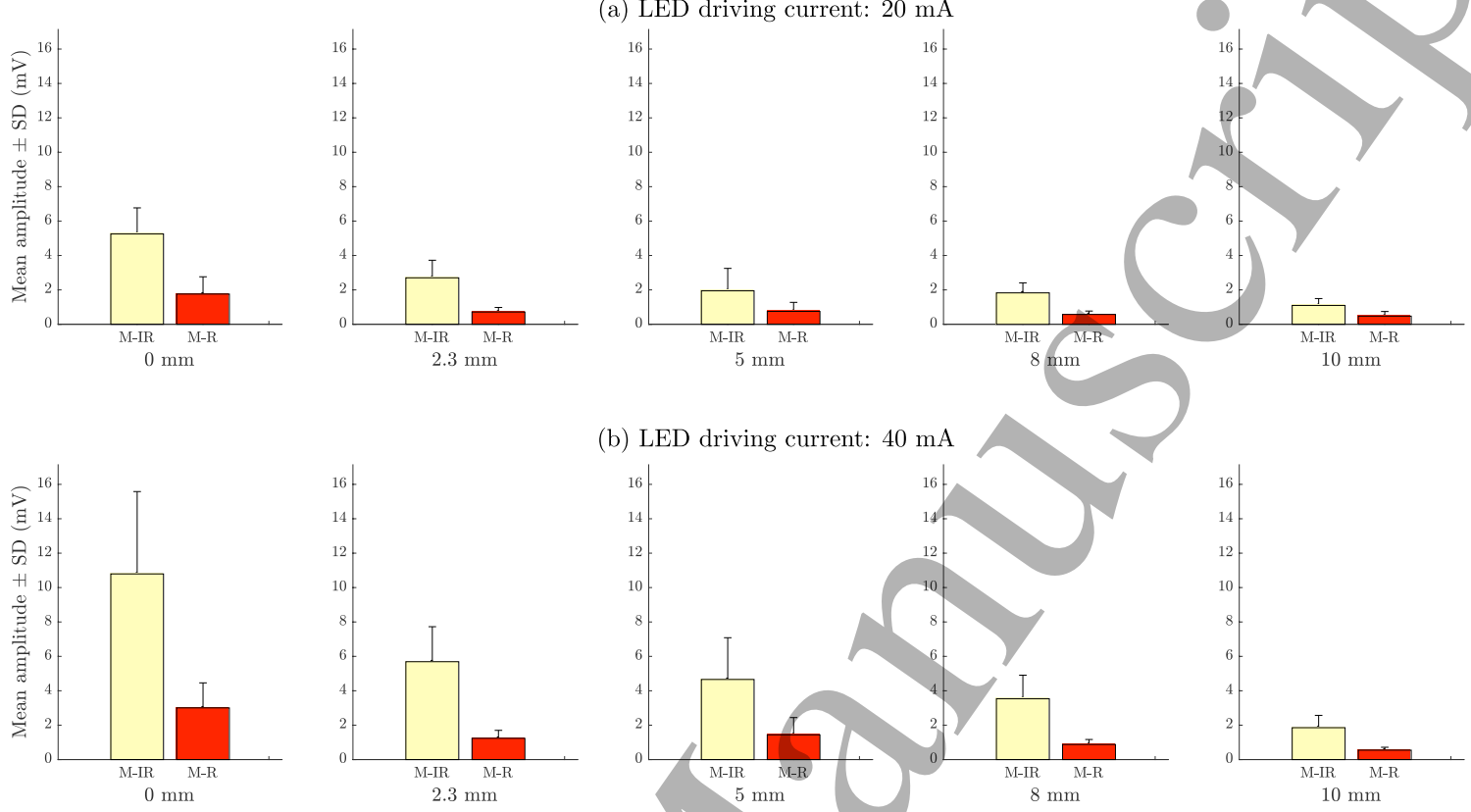

(b) LED driving current: $40 \mathrm{~mA}$
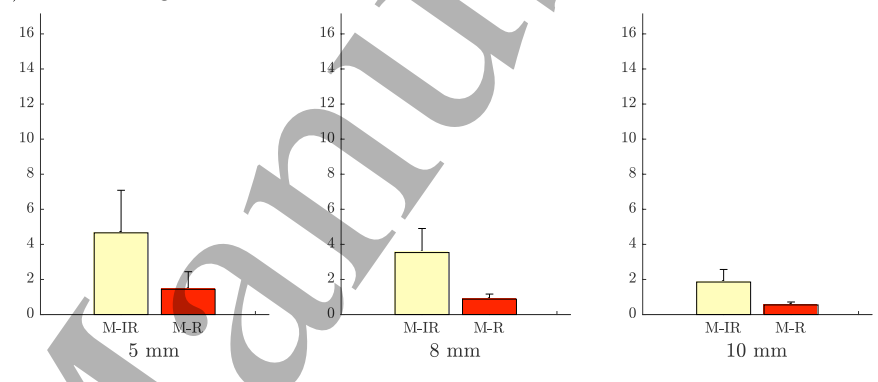

Figure 7: Overall mean and standard deviation of the ac PPG amplitudes $\left(\bar{M}_{a c} \pm \overline{S D}_{a c}\right)$ at different sensor-buccal distances. The results present the overall mean and standard deviation from the buccal mucosa using red (M-R, red) and infrared (M-IR, yellow) wavelengths at two light intensities by driving the LEDs with currents of (a) $20 \mathrm{~mA}$ and (b) $40 \mathrm{~mA}$.

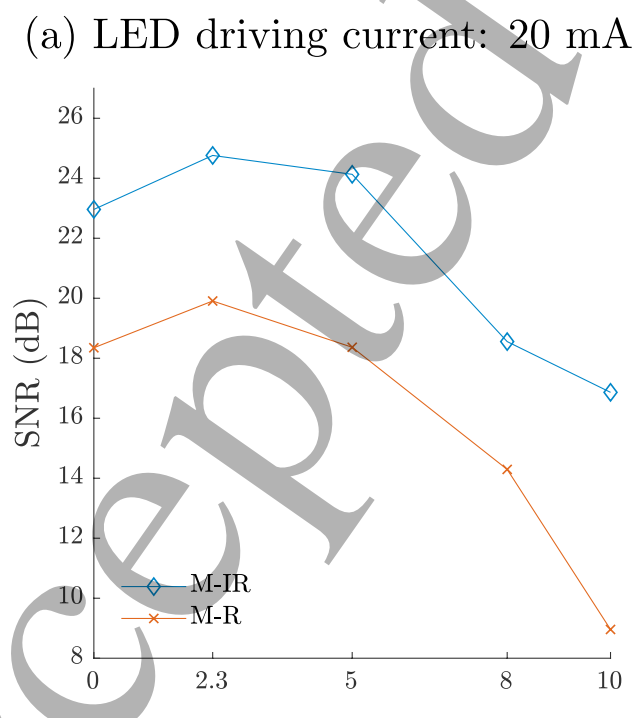

(b) LED driving current: $40 \mathrm{~mA}$

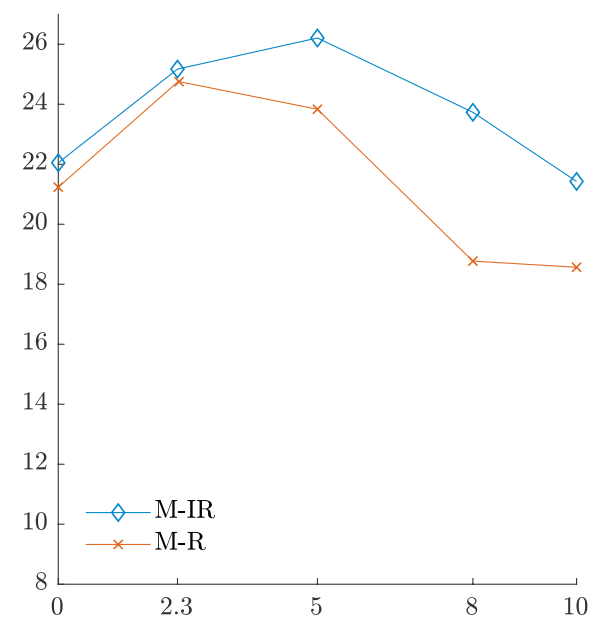

Figure 8: Overall mean of the signal-to-noise ratio $\left(\bar{M}_{S N R}\right)$ for red (M-R SNR, orange $\left.-\times-\right)$ and infrared (M-IR SNR, blue $-\diamond-$ ) at different sensor-buccal separations. Signal-to-noise ratio was calculated for each LED driving current, (a) $20 \mathrm{~mA}$; (b) $40 \mathrm{~mA}$. 


\section{Discussion}

Photoplethysmography signals were obtained at two light intensities, driven with currents of $20 \mathrm{~mA}$ and $40 \mathrm{~mA}$, after altering the distance between the intraluminal sensor and the buccal mucosa, figure 6 . As expected, the PPG amplitude and morphology differed with the changes in the separation distance between the intraluminal optical sensor and the buccal mucosal tissue. The overall trend displayed a decrease in the PPG amplitude as the sensor-buccal separation increased.

Similar trends were observed when comparing the $\bar{M}_{a c}$ between the infrared and red PPG signals, as shown in figure 7. For the infrared LED, with a driving current of $20 \mathrm{~mA}$, the ANOVA test indicated statistical significance within the five $\bar{M}_{a c}$ obtained at each distance $[F=41.025, p=0.001$ $(<0.05)$ ]. Results from the Bonferroni correction, table 2, showed the multiple comparison of sensorbuccal distance of $0 \mathrm{~mm}$ against the remaining distances, having significant differences at $5 \%$ level between the overall mean of infrared ac PPG amplitudes from the buccal mucosa at $0 \mathrm{~mm}\left(\bar{M}_{a c}=5.25 \mathrm{mV}\right)$ compared to the overall means of infrared ac PPG amplitudes at $2.3 \mathrm{~mm}, 5 \mathrm{~mm}, 8 \mathrm{~mm}$ and $10 \mathrm{~mm}$ $\left(\bar{M}_{a c}=2.70 \mathrm{mV}, 1.95 \mathrm{mV}, 1.83 \mathrm{mV}\right.$, and $1.09 \mathrm{mV}$ respectively).

The detected red ac amplitudes were smaller than the infrared ac amplitudes. This is a common finding due to the differences in the light absorption of oxygenated and deoxygenated haemoglobin at these two wavelengths (Kyriacou, 2006; Webster, 1997). For the red LED, with a driving current of $20 \mathrm{~mA}$, the ANOVA test indicated statistical significance within the five $\bar{M}_{a c}$ obtained at each distance $[F=16.516, p=0.001(<0.05)]$. The Bonferroni correction showed there were significant differences between the buccal distance of $0 \mathrm{~mm}$ against the remaining distances at a $5 \%$ level between the overall mean of red ac PPG amplitudes from the buccal mucosa at $0 \mathrm{~mm}\left(\bar{M}_{a c}=1.76 \mathrm{~mm}\right)$ compared to the means of red ac PPG amplitudes at $2.3 \mathrm{~mm}, 5 \mathrm{~mm}, 8 \mathrm{~mm}$ and $10 \mathrm{~mm}\left(\bar{M}_{a c}=0.75 \mathrm{mV}, 0.72 \mathrm{mV}, 0.57 \mathrm{mV}\right.$, and $0.47 \mathrm{mV}$ respectively). The overall means of red ac PPG amplitudes were found to have no statistical difference between the sensor-buccal distance of $2.3 \mathrm{~mm}$ and all distances up to $10 \mathrm{~mm}$ at a $5 \%$ level.

At a driving current of $40 \mathrm{~mA}$, observations from table 2 suggests more pairs of different distances showing a significant difference between the $\bar{M}_{a c}$ 's. By increasing the LED driving currents from $20 \mathrm{~mA}$ to $40 \mathrm{~mA}$, the light intensity increases by two folds, resulting in the $\bar{M}_{a c}$ for both wavelengths to approximately double. For instance, at $0 \mathrm{~mm}$ (contact), the $\bar{M}_{a c}$ for infrared was found to be $5.25 \mathrm{mV}$ and 10.79 $\mathrm{mV}$, respectively. The increase in amplitude is due to the increase in the number of photons emitted in order to penetrate the tissue; similarly increasing the number of returning photons to the photodetector, therefore resulting in a larger PPG amplitude.

Referring to figure 8 (a), high SNRs were observed up to distances of $5 \mathrm{~mm}$, as exceeding this distance lead to a substantial reduction in the red SNR, from approximately $18 \mathrm{~dB}$ to $8.5 \mathrm{~dB}$. As the sensor-buccal separation increased, the PPG signals detected at both LED driving currents became of poor quality, which are identifiable due to the erratic and slight resemblance of the conventional signals, as shown in figure $6(\mathrm{c})$. As the optical sensor-tissue separation distance increases, the photons reaching the mucosal lining diminishes, resulting in the reduction of photons penetrating the tissue. The number of photons returning to the photodiode could also be compromised by the physical distance of the optics and the buccal mucosa. As the number of photons returning to the photodiode is significant to the quality of PPG 
signals, the use of $40 \mathrm{~mA}$ within the intestine would be chosen. Referring to figure 8(b), with the increase of emitted photons, the overall SNR for both wavelengths were seen to increase and approximately halve the SNR range.

Preliminary blood oxygen saturation estimations were conducted as an indication whether the system had the capability of calculating reliable $S_{p} \mathrm{O}_{2}$ values when an optical sensor-tissue separation was present. Considering the left index finger $S_{p} O_{2}$ at $0 \mathrm{~mm}$ (contact) as the reference value, table 1 shows an agreement between the estimated saturations obtained from the buccal mucosa and finger. The t-test suggests no significant differences between the $\bar{M}_{S_{p} O_{2}}$ buccal mucosa and finger at each distance, where p-values above 0.05 were found. As an observation, when the optical sensor is at a farther distance, there are slight reductions in the estimated $\bar{M}_{S_{p} O_{2}}$ values and an increase in the $\overline{S D}_{S_{p} O_{2}}$. This is due to the inaccurate determination of the ratio of ratios (equation 2), which relates to the diminishing SNR.

Discrepancies in the results for the separation distance of $0 \mathrm{~mm}$ (contact) was due to a challenge encountered by the stabilisation of the intraluminal sensor within the mouth. As measurements during contact were obtained with a plastic sheath, each individual/handled the sensor differently. Furthermore, assumptions were made for the SNR to decrease proportionally as the separation distance increased. However, due to participants swallowing and the resultant electromyogram interference from the cheek, recorded signals were hindered.

Despite the challenges encountered, the study provides confidence and reassurance that the sensor would obtain good quality PPG signals from an endocavity; even when a sensor-tissue separation may occur. With medical reference to the intestine anatomy, it is known for the intestine to be in a collapsed state (Nicholls and Dozois, 1997), which supports the assumption of the sensor not exceeding a separation distance of $5 \mathrm{~mm}$. Consequently, a higher light intensity such as $40 \mathrm{~mA}$, would guarantee the capability of acquiring reliable PPG signals and $S_{p} \mathrm{O}_{2}$ estimations to aid in the dynamic monitoring of intestinal viability via an intraluminal route.

\section{Conclusion}

The dual wavelength, reflectance, intraluminal PPG sensor was developed with the long-term aim of dynamically assessing intestinal viability continuously, during intestinal resectional surgery for cancer and benign conditions. Utilising the human buccal mucosa as a surrogate of the intestinal mucosa, the study demonstrated feasible acquisition of PPG signals. Furthermore, good quality PPG signals could be acquired for up to a $5 \mathrm{~mm}$ sensor-tissue separation. Within this range, PPG signals of high quality and SNR were produced, thereby providing reliable $S_{p} O_{2}$ estimations. This preliminary study suggests the ability to obtain PPG signals within the lumen of the large intestine, to assess intestinal viability. The evaluations for the current study was carried out in a controlled environment, and repeating the study in a clinical setting may result in different findings, which may be a limitation of our study. Further experiments are required to study the potential impact of intestinal contents, both liquids and solids on the quality of the signals acquired from within the intestinal lumen. Clinical, proof of concept studies are being planned to test the novel intraluminal intestinal optical sensor as a tool to monitor intestinal viability during and after surgery. 


\section{References}

Abdollahi, Z., Thaha, M. A., Kyriacou, P. A. and Phillips, J. P. (2015), 'Performance of a novel optical sensor for intraoperative assessment of intestinal viability -'proof of principle study", Gut 64(June).

Allen, J. (2007), 'Photoplethysmography and its application in clinical physiological measurement.', Physiol. Meas. 28(3), R1-R39.

Budidha, K., Rybynok, V. and Kyriacou, P. A. (2018), 'Design and Development of a Modular, Multichannel Photoplethysmography System', IEEE Trans. Instrum. Meas. pp. 1-12.

Cancer Research, U. (2016), 'Bowel cancer incidence statistics'.

URL: http://www.cancerresearchuk.org/health-professional/cancer-statistics

Hickey, M. and Kyriacou, P. A. (2007), 'Optimal spacing between transmitting and receiving optical fibres in reflectance pulse oximetry', J. Phys. Conf. Ser. 85, 012030:148.

Hickey, M., Samuels, N., Langford, R. M. and Kyriacou, P. A. (2010),'"Measurement of splanchnic photoplethysmographic signals using a new reflectance fiber optic sensor', J. Biomed. Opt. 15(2), 027012:1-8.

Hickey, M., Samuels, N., Randive, N., Langford, R. M. and Kyriacou, P. A. (2011), 'An in vivo investigation of photoplethysmographic signals and preliminary pulse oximetry estimation from the bowel using a new fiberoptic sensor', Anesth. Analg. 112(5), 1104-1109.

Hogg, R. V. and Ledolter, J. (1987), Engineering Statistics, MacMillan Publishing Company.

Hyman, N. H. (2009), 'Managing anastomotic leakss from intestinal anastomoses.', Surgeon 7(1), 31-35.

Jafari, M. D., Lee, K. H., Halabi, W. J., Mills, S. D., Carmichael, J. C., Stamos, M. J. and Pigazzi, A. (2013), 'The use of indocyanine green fluorescence to assess anastomotic perfusion during robotic assisted laparoscopic rectal surgery', Surg. Endosc. 27(8), 3003-3008.

Jafari, M. D., Wexner, S. D., Martz, J. E., McLemore, E. C., Margolin, D. a., Sherwinter, D. a., Lee, S. W., Senagore, A. J., Phelan, M. J. and Stamos, M. J. (2015), 'Perfusion assessment in laparoscopic left-sided/anterior resection (PILLAR II): A multi-institutional study.', J. Am. Coll. Surg. 220(1), 8292.e1.

Kingham, T. P., Pachter, H. L., Brisinda, G., Vanella, S., Cadeddu, F. and Mazzeo, P. (2009), 'Colonic Anastomotic Leak: Risk Factors, Diagnosis, and Treatment', J. Am. Coll. Surg. 208(2), 269-278.

Kyriacou, P. A. (2006), 'Pulse oximetry in the oesophagus', Physiol. Meas. 27(1), R1-R35.

Kyriacou, P. A. (2013), 'Direct pulse oximetry within the esophagus, on the surface of abdominal viscera, and on free flaps', Anesth. Analg. 117(4), 824-833.

Lee, E. S., Bass, A., Arko, F. R., Heikkinen, M., Harris, E. J., Zarins, C. K., van der Starre, P. and Olcott, C. (2006), 'Intraoperative Colon Mucosal Oxygen Saturation During Aortic Surgery', J. Surg. Res. 136(1), 19-24. 
Massey, F. (1951), 'The Kolmogorov-Smirnov Test for Goodness of Fit', J. Am. Stat. Assoc. 46(253), 6878.

Mitra, S. K. (2001), Digital signal processing, 2 edn, McGraw-Hill, New York.

Nachiappan, S., Askari, A., Currie, A., Kennedy, R. H. and Faiz, O. (2014), 'Intraoperative assessment of colorectal anastomotic integrity: A systematic review', Surgical Endoscopy 28(9), 2513-2530.

Neter, J., Kutner, M. H., Nachtsheim, C. J. and Wasserman, W. (1996), Applied Linear Statistical Models, 4 edn, Irwin Press.

Nicholls, R. J. and Dozois, R. R. (1997), Surgery of the colon \& rectum, 1 edn, Churchill Livingstone, New York.

Patel, Z., Thaha, M. and Kyriacou, P. (2017), 'Development of an intraluminal intestinal photoplethysmography sensor', in 'Proceedings of the Annual International Conference of the IEEE Engineering in Medicine and Biology Society, EMBS', IEEE, Seogwipo, South Korea, pp. 1840-1843.

Sun, Y. and Thakor, N. (2016), 'Photoplethysmógraphy Revisited: From Contact to Noncontact, from Point to Imaging', IEEE Trans. Biomed. Eng. 63(3), 463-477.

Webster, J. (1997), Design of Pulse Oximeters, 1 edn, Taylor \& Francis Ltd. 